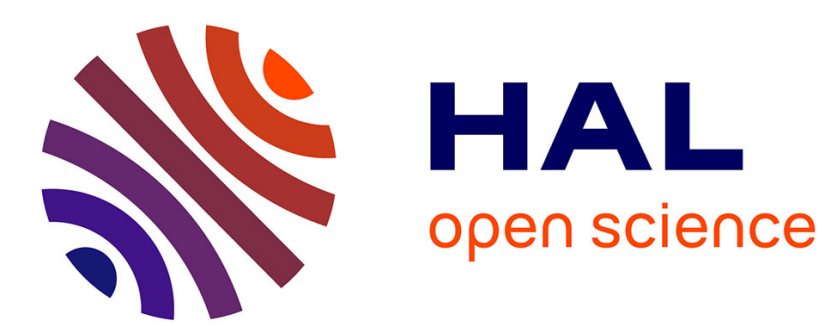

\title{
Curvature Dependence of the Liquid-Vapor Surface Tension beyond the Tolman Approximation
}

Nicolas Bruot, Frédéric Caupin

\section{To cite this version:}

Nicolas Bruot, Frédéric Caupin. Curvature Dependence of the Liquid-Vapor Surface Tension beyond the Tolman Approximation. Physical Review Letters, 2016, 116, pp.056102. 10.1103/PhysRevLett.116.056102 . hal-02303905

\section{HAL Id: hal-02303905 \\ https://univ-lyon1.hal.science/hal-02303905}

Submitted on 25 Jan 2021

HAL is a multi-disciplinary open access archive for the deposit and dissemination of scientific research documents, whether they are published or not. The documents may come from teaching and research institutions in France or abroad, or from public or private research centers.
L'archive ouverte pluridisciplinaire HAL, est destinée au dépôt et à la diffusion de documents scientifiques de niveau recherche, publiés ou non, émanant des établissements d'enseignement et de recherche français ou étrangers, des laboratoires publics ou privés. 


\title{
Curvature Dependence of the Liquid-Vapor Surface Tension beyond the Tolman Approximation
}

\author{
Nicolas Bruot and Frédéric Caupin \\ Institut Lumière Matière, UMR5306 Université Claude Bernard Lyon 1-CNRS, Université de Lyon, \\ Institut Universitaire de France, 69622 Villeurbanne cedex, France \\ (Received 4 September 2015; revised manuscript received 30 November 2015; published 2 February 2016)
}

\begin{abstract}
Surface tension is a macroscopic manifestation of the cohesion of matter, and its value $\sigma_{\infty}$ is readily measured for a flat liquid-vapor interface. For interfaces with a small radius of curvature $R$, the surface tension might differ from $\sigma_{\infty}$. The Tolman equation, $\sigma(R)=\sigma_{\infty} /(1+2 \delta / R)$, with $\delta$ a constant length, is commonly used to describe nanoscale phenomena such as nucleation. Here we report experiments on nucleation of bubbles in ethanol and $n$-heptane, and their analysis in combination with their counterparts for the nucleation of droplets in supersaturated vapors, and with water data. We show that neither a constant surface tension nor the Tolman equation can consistently describe the data. We also investigate a model including $1 / R$ and $1 / R^{2}$ terms in $\sigma(R)$. We describe a general procedure to obtain the coefficients of these terms from detailed nucleation experiments. This work explains the conflicting values obtained for the Tolman length in previous analyses, and suggests directions for future work.
\end{abstract}

DOI: 10.1103/PhysRevLett.116.056102

Nucleation in metastable phases is a ubiquitous phenomenon, relevant to important fields such as atmospheric research [1], mechanics of plants and trees [2-4], and in the chemical industry to avoid vapor explosion accidents (or "spill accidents") [5]. The nucleation rate is exquisitely sensitive to the value of the surface tension $\sigma$ between the metastable and the stable phase. As the size of the critical nucleus that triggers the phase change is in the nanometer range, the value of $\sigma$ relevant to nucleation may differ from the bulk one. The idea of a dependence of the surface tension on the curvature of the interface between phases has been studied by Tolman [6], who proposed for a spherical droplet with radius $R$

$$
\frac{\sigma_{\infty}}{\sigma(R)}=1+\frac{2 \delta_{\infty}}{R},
$$

where $\delta_{\infty}$ is the Tolman length. Determining in experiments $\sigma(R)$ is critical to developing accurate nucleation theories. It can also serve as an input to validity checks of density functional theory calculations and numerical simulations. More generally, the small scale limit at which macroscopic laws break down is an active field of research, as shown for instance by recent studies on vapor pressure of nanodroplets [7], or on flows in nanochannels [8], which are of crucial importance for oil recovery and catalysis.

The curvature dependence of surface tension has been mainly studied theoretically and numerically, with conflicting results about the magnitude and even the sign of the effect $[6,7,9-18]$. The dearth of experimental data stems from the difficulties inherent to measurements on nanoscopic objects. In this work we circumvent this problem by use of the nucleation theorem (NT) $[19,20]$, which allows obtaining information on the nanoscopic critical nucleus from a macroscopic observable, the nucleation rate (number of nucleation events per unit volume and time). In addition, we adopt a comprehensive approach, treating on the same footing the two symmetric cases of nanodroplets (related to the nucleation of a liquid from a supersaturated vapor, condensation) and nanobubbles (related to the nucleation of a vapor in a metastable liquid, cavitation). To complement existing data on condensation, we have performed acoustic cavitation experiments on ethanol and $n$-heptane.

In acoustic cavitation, the liquids are stretched using a few cycles of a focused acoustic wave at $1 \mathrm{MHz}$ to trigger nucleation (see Ref. [21] and the Supplemental Material [22] for details). The wave frequency sets the experimental time and volume, and, consequently, the observable nucleation rate. The pressure at which this rate is reached is shown in Fig. 1(a) for ethanol and in Fig. S1(a) [22] for heptane. Compared to a previous study where the cavitation pressures were based on an indirect estimate [23], we have now measured them directly with a fiber-optic probe hydrophone (FOPH) [24]. These more accurate measurements lead to lower pressures than the previous study, as expected because of the nonlinearities in the acoustic wave (see the Supplemental Material [22] and Refs. [25,26]).

An excellent introduction to the concept of curvaturedependent surface tension can be found in Ref. [10]. We just introduce here the relevant quantities on the example of condensation. Consider a small spherical droplet of liquid in equilibrium with its supersaturated vapor at chemical potential $\mu$. The pressure of the bulk liquid and vapor at $\mu$ are $P_{L}$ and $P_{V}$, and their densities $\rho_{\mathrm{L}}$ and $\rho_{\mathrm{V}}$, respectively. The key point is that the surface tension $\sigma$ depends on the radius $R$ chosen for the dividing sphere which separates by 

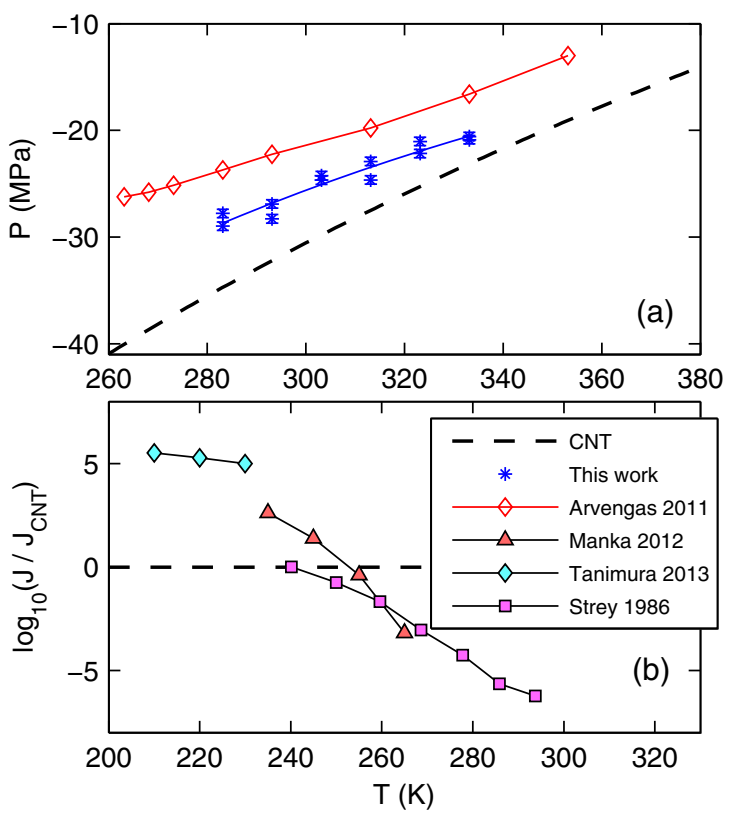

FIG. 1. Comparison of nucleation pressures or rates (markers) with $\mathrm{CNT}_{0}$ (dashed lines) in ethanol. (a) Acoustic cavitation pressures obtained with the FOPH (blue stars) are compared to previous pressure estimates (red diamonds) via a static pressure method [23]. The new, more accurate points are consistently more negative, as expected (see the Supplemental Material [22]). The blue line is a guide to the eye. (b) Condensation data. Each graph represents the logarithm of the ratio between the nucleation rate and the $\mathrm{CNT}_{0}$ prediction. The data sources are indicated in the legend. See Fig. S1 [22] for the corresponding graphs for heptane and water.

convention the liquid and vapor regions. Two radii are of particular interest in describing the droplet. The first is $R_{e}$, the radius of the equimolar dividing surface. The second is $R_{s}$, the radius of the sphere at which the Laplace relation is fulfilled:

$$
\Delta P=P_{L}-P_{V}=\frac{2 \sigma_{s}}{R_{s}},
$$

where $\sigma_{s}=\sigma\left(R_{s}\right) \cdot R_{s}$ allows us to write the energy barrier for nucleation $\Delta \Omega$ in a compact form:

$$
\Delta \Omega=\frac{4 \pi}{3} R_{s}^{2} \sigma_{s}=\frac{16 \pi \sigma_{s}^{3}}{3 \Delta P^{2}} .
$$

In classical nucleation theory (CNT), the nucleation rate $J$ for the phase change is

$$
J=J_{0} \exp \left(-\frac{\Delta \Omega}{k_{B} T}\right)
$$

with $J_{0}$ a prefactor, whose expression is given in the Supplemental Material [22]. The knowledge of $J$ at a given $\mu$ or $\Delta P$ (the quantity controlled in an experiment) thus gives access to $\Delta \Omega$ from Eq. (4), $\sigma_{s}$ from Eq. (3) and $R_{s}^{*}$ from Eq. (2) Starred quantities are relative to the critical nucleus, at which the energy barrier $\Delta \Omega$ is reached. In addition, experiments can give access to $R_{e}^{*}$. Indeed, if the dependence of $J$ on $\mu$ is known, the excess number of molecules in the critical nucleus is $[19,20]$

$$
\Delta n^{*}=k_{B} T\left(\frac{\partial \ln \left(J / J_{0}\right)}{\partial \mu}\right)_{T} .
$$

For $\rho_{V} \ll \rho_{L}$, and assuming a spherical critical droplet whose density at the center reaches the bulk value, this leads to an expression for the volume of the sphere with radius $R_{e}^{*}$ (see the Supplemental Material [22]):

$$
V_{e}^{*}=\frac{4 \pi}{3} R_{e}^{3}=\frac{\left|\Delta n^{*}\right|}{\rho_{L}}=\frac{k_{B} T}{\rho_{L}}\left|\left(\frac{\partial \ln \left(J / J_{0}\right)}{\partial \mu}\right)_{T}\right| .
$$

Equations (3) to (6) hold for both cavitation and condensation, provided that adequate expressions for $\Delta P, \mu$, and $J_{0}$ are used.

To test different models for the surface tension, we have used experimental values of $J$ at known $\mu$ (condensation data), or, equivalently, $\Delta P$ at known $J$ (cavitation data), and $V_{\mathrm{e}}^{*}$. The models are described below and are summarized in Table I.

In the standard version of the CNT (Chap. 3 of Ref. [5]), $\mathrm{CNT}_{0}, \sigma_{s}$ is assumed to always remain equal to the value for a planar interface $\sigma_{\infty}$, which is equivalent to setting $R_{e}=R_{s}$, and $\Delta \Omega=16 \pi \sigma_{\infty}^{3} /\left(3 \Delta P^{2}\right)$. $\mathrm{CNT}_{0}$ is notorious to fail in predicting correct nucleation rates, for cavitation [27] as well as for condensation [14,28-35]. This appears clearly in Fig. 1 and Fig. S1 [22], where the FOPH experiments are plotted along with condensation data $[28,31,32,36-40]$, and with a cavitation point from a water inclusion in quartz [27]. For instance, Fig. 1(b), Figs. S1(b) and S1(d) [22] highlight for the three fluids a crossover temperature below which $\mathrm{CNT}_{0}$ underestimates the condensation rates, and above which they are overestimated. This crossover had already been observed in single data sets for ethanol and water condensation, e.g., Refs. [32] and

TABLE I. Summary of the models tested in this Letter. For each model, we indicate the expression for the surface tension, the free parameters, and the experimental data used to extract these parameters.

\begin{tabular}{lccc}
\hline \hline Model & Surface tension & Free parameters & Input data \\
\hline $\mathrm{CNT}_{0}$ & $\sigma\left(R_{s}\right)=\sigma_{\infty}$ & None & None \\
$\mathrm{CNT}_{1}$ & $\sigma_{\infty} / \sigma\left(R_{s}\right)=1+\left(2 \delta_{\infty} / R_{s}\right)$ & $\delta_{\infty}$ & $J$ \\
$\mathrm{CNT}_{2}$ & $\sigma_{\infty} / \sigma\left(R_{s}\right)=1+\left(2 \delta_{\infty} / R_{s}\right)+\left(\delta_{\infty}^{2}+\alpha\right) / R_{s}^{2}$ & $\delta_{\infty}$ and $\delta_{\infty}^{2}+\alpha$ & $J$ and $V_{e}^{*}$ \\
\hline \hline
\end{tabular}


[41], and the combination of several sets makes this conclusion stronger.

We therefore investigate other models with $R_{e} \neq R_{s}$. We use a functional form suggested by simulations [10]:

$$
\delta\left(R_{s}\right)=\delta_{\infty}+\frac{\alpha}{R_{s}} .
$$

Starting with a first variant of $\mathrm{CNT}, \mathrm{CNT}_{1}$, which assumes $\alpha=0$ in Eq. (7), it can be shown that [10]

$$
\frac{\sigma_{\infty}}{\sigma_{s}}=1+\frac{2 \delta_{\infty}}{R_{s}}+\mathcal{O}\left(\frac{1}{R_{s}^{2}}\right),
$$

which is similar to Eq. (1). One can then calculate $\delta_{\infty}$ from the experimental nucleation rates (see the Supplemental Material [22]). The analysis usually stops there, which does not provide a full test of $\mathrm{CNT}_{1}$. We take a step further, and predict $V_{e}^{*}$ from $\delta_{\infty}$ with $\mathrm{CNT}_{1}$ (see the Supplemental Material [22]). A comparison between predicted $V_{e}^{*}$ and $V_{e}^{*}$ deduced from the experiments with the NT Eq. (6) becomes possible. To our knowledge, this type of reasoning has been employed only for water [27,31], with a seemingly satisfactory agreement. By a more comprehensive analysis of all the data sets gathered for ethanol, heptane, and water, covering a broader range for temperature and degree of metastability, we find discrepancies that reveal the actual failure of $\mathrm{CNT}_{1}$. Figure 2 and Fig. S2 [22] show $\delta_{\infty}$ calculated from the cavitation and condensation experiments. Quantities relative to cavitation and condensation are labelled by cav or cond, respectively. If $\mathrm{CNT}_{1}$ were valid, we would expect to find that $\delta_{\infty}^{\text {cav }}$ and $\delta_{\infty}^{\text {cond }}$ do not depend on $R_{s}^{*}$ and that $\delta_{\infty}^{\text {cav }}=-\delta_{\infty}^{\text {cond }}$ for the same temperatures [10]. For each of the fluids, the points do not collapse on a single curve, as indicated by the double arrows, even when taking into account the experimental uncertainties (see the Supplemental Material [22] for details). This suggests that $\delta_{\infty}$ does in fact depend on $R_{s}^{*}$. We emphasize that the disagreement can usually not be seen when looking at the data of a single condensation experiment. This is because various independent supersaturation and temperature values are needed to conclude and the combination of several condensation and cavitation experiments extends the range of both parameters. We mentioned above a crossover temperature when comparing experimental and $\mathrm{CNT}_{0}$ condensation rates. It translates here into a change of $\delta_{\text {cond }}$ from positive to negative values when $T$ increases (Fig. 2 and Fig. S2 [22]). This behavior is not in support of $\mathrm{CNT}_{1}$. The situation gets even worse when comparing in Fig. 3 and Fig. S3 $V_{e}^{*}$ extracted from this model (small, light markers) with $V_{e}^{*}$ obtained using the NT (big, dark markers). Overall, these agree at low temperature, but strongly disagree above $250-300 \mathrm{~K}$ depending on the fluid. We note that the approximations needed to deduce $V_{e}^{*}$ from experiments with Eq. (6) lead to an underestimate of $V_{e}^{*}$ for both cavitation and condensation, so that the disagreement with $\mathrm{CNT}_{1}$ can be only stronger than shown

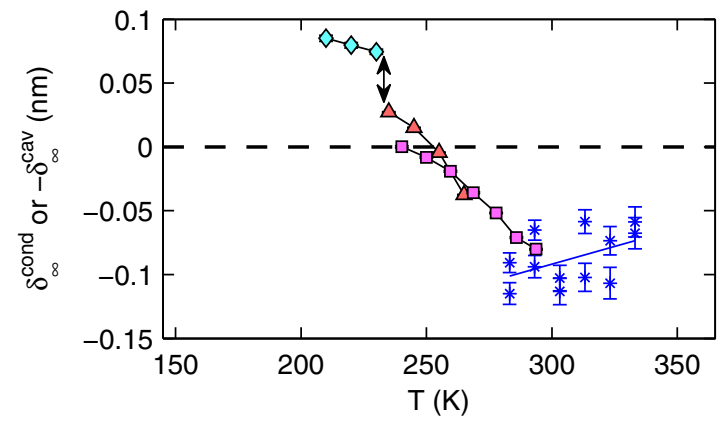

FIG. 2. $\delta_{\infty}$ depending on temperature for cavitation (star markers) and condensation (filled markers) in ethanol. The legend is the same as in Fig. 1. The double arrow points to discrepancies that suggest the failure of the $\mathrm{CNT}_{1}$ model: $\delta_{\infty}$, which is expected to be the Tolman length, would not only depend on $T$ but also on $\mu$. The blue line is a guide to the eye of the FOPH data. See Fig. S2 [22] for heptane and water.

in Fig. 3 and Fig. S3 [22]. Also, the critical volumes for cavitation may display a systematic error bigger than the statistical error bars shown here because of an extrapolation in the data analysis. All these details are investigated in the Supplemental Material [22] and Refs. [42,43] and do not change any of our conclusions.

We now move on to a second variant of $\mathrm{CNT}, \mathrm{CNT}_{2}$, based on Eq. (7) with two parameters $\delta_{\infty}$ and $\alpha \neq 0$. It can then be shown that [44]:

$$
\frac{\sigma_{\infty}}{\sigma_{s}}=1+\frac{2 \delta_{\infty}}{R_{s}}+\frac{\delta_{\infty}{ }^{2}+\alpha}{R_{s}{ }^{2}}+\mathcal{O}\left(\frac{1}{R_{s}{ }^{3}}\right)
$$

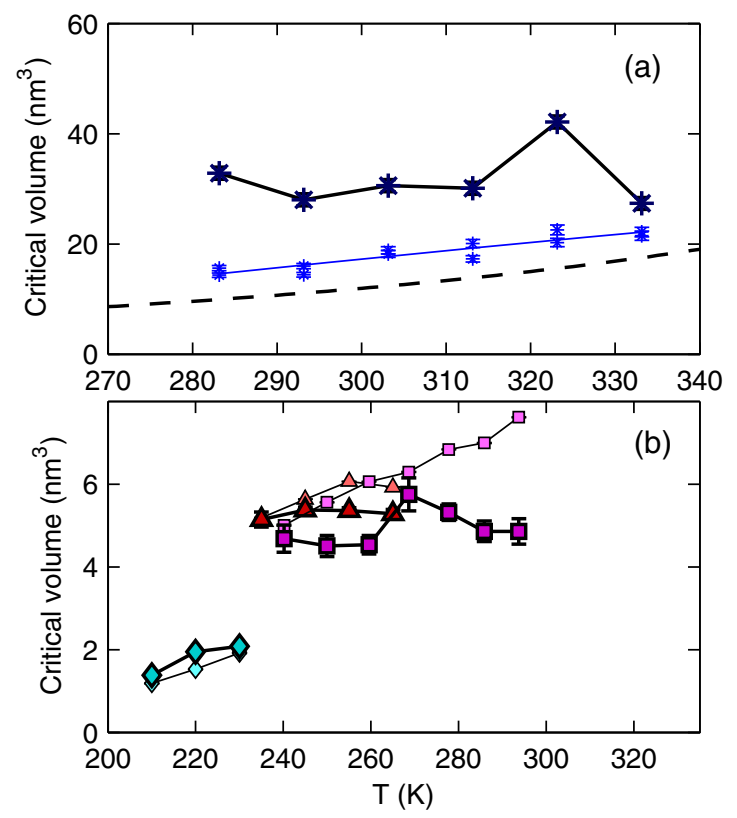

FIG. 3. Critical volumes in (a) cavitation and (b) condensation experiments for ethanol. The thick and thin markers represent the volumes from the $\mathrm{NT}$ and from $\mathrm{CNT}_{1}$, respectively. The legend is the same as in Fig. 1. See Fig. S3 [22] for heptane and water. 
It may seem that adding an extra parameter would automatically allow a better fitting of the data. But we also use more experimental input (see Table I). For $\mathrm{CNT}_{1}$, we used only the experimental nucleation rates to calculate $\delta_{\infty}$, and compared the $\mathrm{CNT}_{1}$ prediction for $V_{e}^{*}$ with the values from experiments on which the nucleation theorem can be applied. For $\mathrm{CNT}_{2}$, we use both the nucleation rates and $V_{e}^{*}$ from those experiments to directly calculate $\delta_{\infty}$ and $\delta_{\infty}^{2}+\alpha$ (see the Supplemental Material [22]). The success of the approach must therefore be assessed by checking if the various data sets lead to master curves for both $\delta_{\infty}$ and $\delta_{\infty}^{2}+\alpha$ as a function of the temperature. The results are plotted in Fig. 4 and Fig. S4 [22]. We have omitted here the FOPH data, since, unfortunately, the corresponding error bars on $\delta_{\infty}$ and $\delta_{\infty}^{2}+\alpha$ would be of the order of the size of the $y$ axes (see the Supplemental Material [22]). For heptane, only one data set is available and does not allow us to test the data collapse. For the other fluids, we observe a more consistent description of the data (see, in particular, the improvement of the discrepancy between the Tanimura 2013 and the Manka 2012 data compared to Fig. 2). The agreement is still not perfect, even when taking into account the statistical uncertainties on the points. This is possibly indicative of systematic errors specific to the different experiments, or of some limitation of the theory, such as that of a spherical critical nucleus. Compared to $\mathrm{CNT}_{1}, \mathrm{CNT}_{2}$ yields a Tolman length $\delta_{\infty}$ with a weaker temperature dependence, and for ethanol and water $\delta_{\infty}$ now keeps a positive sign. For heptane, $\delta_{\infty}$ is close to zero, maybe slightly negative. For the three fluids, we noted that the second order term $\left(\delta_{\infty}^{2}+\alpha\right) /\left(R_{\mathrm{s}}^{*}\right)^{2}$ is often of the same

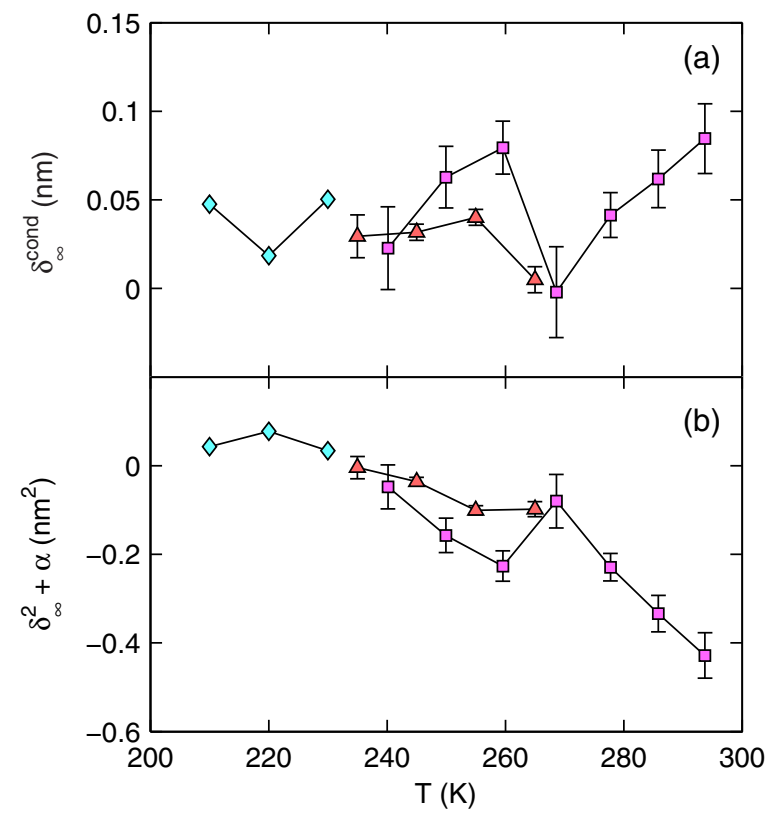

FIG. 4. $\mathrm{CNT}_{2}$ parameters $\delta_{\infty}$ (a) and $\delta_{\infty}^{2}+\alpha$ (b) derived from condensation experiments. See Fig. 1 for the legend, and Fig. S4 [22] for heptane and water. order of magnitude as the first order term $\delta_{\infty} / R_{\mathrm{S}}^{*}$ (see the Supplemental Material [22]).

Our results show that, at least for ethanol, heptane, and water, the usual Tolman equation Eq. (1) is not enough to properly describe experiments. Therefore, attempts to analyze experiments with $\mathrm{CNT}_{1}$ (such as in Refs. [27,31]) may yield inaccurate determinations of $\delta_{\infty}$, and this study partly explains the confusion in the long-standing debate on the sign of the Tolman length [45]. The $\mathrm{CNT}_{2}$ approach seems to give more consistent results.

A variety of simulations have been performed and motivated our work. We have tried to compare the experiments to these. For heptane, the $\delta_{\infty}^{\text {cond }}$ we found is close to zero and possibly negative (around $-0.02 \mathrm{~nm}$ ). An expression of the Tolman length as a function of the isothermal compressibility $\kappa_{T}$ has been proposed [46,47]: $\delta_{\infty}=$ $-\kappa_{T} \sigma_{\infty}$. At $265 \mathrm{~K}$, the formula yields $\delta_{\infty} \approx-0.03 \mathrm{~nm}$ for heptane, which is close to the experiments in Fig. S4(a) [22]. Heptane may be crudely approximated by a LennardJones fluid, for which DFT calculations and MC simulations $[9,10]$ seem to point to a slightly negative value for $\delta_{\infty}^{\text {cond }}(-0.07 \mathrm{~nm})$. However, MD simulations [44] find a positive temperature-dependent $-\delta_{\infty}^{\text {cav }}(+0.1 \mathrm{~nm}$ at $265 \mathrm{~K})$. Also, Iwamatsu [48] estimated the Tolman length from the correlation lengths of the liquid and vapor phases, which for heptane translates into $\delta_{\infty}^{\text {cond }} \approx-0.2 \mathrm{~nm}$, which has a larger magnitude than the experimental value. The parameter $\delta_{\infty}^{2}+\alpha$ has been estimated by MD simulations [44] and by DFT [9,49]. Rescaled to heptane, these estimates all lead to a positive $\left(\delta_{\infty}^{2}+\alpha\right) / R_{s}^{2}$ of about 0.4 [44], while the Rudek data set displays mostly negative values: $\left(\delta_{\infty}^{2}+\alpha\right) /$ $R_{e}^{2}=-0.1$ in average in the $250-275 \mathrm{~K}$ range. By identifying Eq. (9) to the Helfrich form of the surface free energy in Ref. [50], we find the average curvature-elastic moduli $2 k_{c}+\bar{k}_{c}=7 \times 10^{-22} \mathrm{~J}$.

For the other fluids, the different experiments partially collapse on master curves, thus supporting the $\mathrm{CNT}_{2}$ model, but they can hardly be compared to simulations or DFT estimates based on the Lennard-Jones potential. For water, we first note that within $\mathrm{CNT}_{2}$, the cavitation [27] and the condensation [40] experiments yield to positive $\delta_{\infty}^{\text {cond }}$ or $-\delta_{\infty}^{\text {cav }}$. This sign is consistent with simulations based on a monoatomic model of water $(\mathrm{mW})$ where a departure from the Kelvin equation is observed at small droplet radii [7]. However, the $\mathrm{CNT}_{2}$ analysis of two simulations with TIP4P/2005 [11,51] would give the opposite sign: $\delta_{\infty}^{\text {cond }}=-0.066 \mathrm{~nm}$ at $300 \mathrm{~K}$ for Ref. [11], which measured directly the radius dependence of the surface free energy of droplets, and $-\delta_{\infty}^{\mathrm{cav}}=$ $-0.067 \mathrm{~nm}$ for the cavitation simulations in Ref. [51], from which we have calculated $\delta_{\infty}$ with the energy barrier and the critical volume (using the data from their $M$ method to estimate $V_{\mathrm{e}}^{*}$ ).

While our conclusions on the inaccuracy of the $\mathrm{CNT}_{1}$ model are unambiguous-we strongly recommend not to 
use the Tolman equation when analyzing nucleation datathey call for further experiments to confirm the $\mathrm{CNT}_{2}$ model. As the vapor supersaturation can be varied over a broad range in experiments on condensation, they should be more appropriate than cavitation. For ethanol, the success of $\mathrm{CNT}_{2}$ is already very promising, and we provide in the Supplemental Material [22] overall fitting parameters for $\delta_{\infty}$ and $\delta_{\infty}^{2}+\alpha$ that can be used to predict the nucleation rate from any condition. For other fluids, our study provides a procedure with which future measurements of nucleation rates and critical volumes can be analyzed.

We acknowledge funding by the ERC under the European FP7 Grant Agreement No. 240113, and by the Agence Nationale de la Recherche Grant No. 09-BLAN0404-01.

*frederic.caupin@univ-lyon1.fr

[1] B. J. Murray, D. O’Sullivan, J. D. Atkinson, and M. E. Webb, Chem. Soc. Rev. 41, 6519 (2012).

[2] Y. Forterre, J. Exp. Bot. 64, 4745 (2013).

[3] X. Noblin, N. O. Rojas, J. Westbrook, C. Llorens, M. Argentina, and J. Dumais, Science 335, 1322 (2012).

[4] T. D. Wheeler and A. D. Stroock, Nature (London) 455, 208 (2008).

[5] P. G. Debenedetti, Metastable Liquids (Princeton University Press, Princeton, NJ, 1996).

[6] R. C. Tolman, J. Chem. Phys. 17, 333 (1949).

[7] M. H. Factorovich, V. Molinero, and D. A. Scherlis, J. Am. Chem. Soc. 136, 4508 (2014).

[8] P. Huber, J. Phys. Condens. Matter 27, 103102 (2015).

[9] B. J. Block, S. K. Das, M. Oettel, P. Virnau, and K. Binder, J. Chem. Phys. 133, 154702 (2010).

[10] A. Tröster, M. Oettel, B. Block, P. Virnau, and K. Binder, J. Chem. Phys. 136, 064709 (2012).

[11] M. N. Joswiak, N. Duff, M. F. Doherty, and B. Peters, J. Phys. Chem. Lett. 4, 4267 (2013).

[12] J. Hrubý, D. G. Labetski, and M. E. H. van Dongen, J. Chem. Phys. 127, 164720 (2007).

[13] T. V. Bykov and X. C. Zeng, J. Chem. Phys. 111, 3705 (1999).

[14] T. P. Bennett and J. C. Barrett, J. Chem. Phys. 137, 124702 (2012).

[15] A. E. van Giessen and E. M. Blokhuis, J. Chem. Phys. 131, 164705 (2009).

[16] M. Horsch, H. Hasse, A. K. Shchekin, A. Agarwal, S. Eckelsbach, J. Vrabec, E. A. Müller, and G. Jackson, Phys. Rev. E 85, 031605 (2012).

[17] E. Santiso and A. Firoozabadi, AIChE J. 52, 311 (2006).

[18] M. P. Moody and P. Attard, Phys. Rev. Lett. 91, 056104 (2003).

[19] D. W. Oxtoby and D. Kashchiev, J. Chem. Phys. 100, 7665 (1994).

[20] D. Kashchiev, J. Chem. Phys. 125, 014502 (2006).

[21] E. Herbert, S. Balibar, and F. Caupin, Phys. Rev. E 74, 041603 (2006).
[22] See Supplemental Material at http://link.aps.org/ supplemental/10.1103/PhysRevLett.116.056102 for materials and methods, results for $n$ heptane and water, formulas for $\mathrm{CNT}_{1}$ and $\mathrm{CNT}_{2}$, and discussion of error bars.

[23] A. Arvengas, E. Herbert, S. Cersoy, K. Davitt, and F. Caupin, J. Phys. Chem. B 115, 14240 (2011).

[24] A. Arvengas, K. Davitt, and F. Caupin, Rev. Sci. Instrum. 82, 034904 (2011).

[25] C. Appert, C. Tenaud, X. Chavanne, S. Balibar, F. Caupin, and D. d'Humiéres, Eur. Phys. J. B 35, 531 (2003).

[26] K. Davitt, E. Rolley, F. Caupin, A. Arvengas, and S. Balibar, J. Chem. Phys. 133, 174507 (2010).

[27] M. E. M. Azouzi, C. Ramboz, J.-F. Lenain, and F. Caupin, Nat. Phys. 9, 38 (2013).

[28] D. Ghosh, D. Bergmann, R. Schwering, J. Wlk, R. Strey, S. Tanimura, and B. E. Wyslouzil, J. Chem. Phys. 132, 024307 (2010).

[29] Y. J. Kim, B. E. Wyslouzil, G. Wilemski, J. Wölk, and R. Strey, J. Phys. Chem. A 108, 4365 (2004).

[30] C. C. M. Luijten, K. J. Bosschaart, and M.E.H. van Dongen, J. Chem. Phys. 106, 8116 (1997).

[31] V. Holten, D. G. Labetski, and M. E. H. van Dongen, J. Chem. Phys. 123, 104505 (2005).

[32] A. A. Manka, J. Wedekind, D. Ghosh, K. Höhler, J. Wölk, and R. Strey, J. Chem. Phys. 137, 054316 (2012).

[33] A.-P. Hyvärinen, H. Lihavainen, Y. Viisanen, and M. Kulmala, J. Chem. Phys. 120, 11621 (2004).

[34] P. Peeters, J. J. H. Gielis, and M. E. H. van Dongen, J. Chem. Phys. 117, 5647 (2002).

[35] J. A. Fisk and J. L. Katz, J. Chem. Phys. 104, 8649 (1996).

[36] S. Tanimura, H. Pathak, and B. E. Wyslouzil, J. Chem. Phys. 139, 174311 (2013).

[37] R. Strey, P. E. Wagner, and T. Schmeling, J. Chem. Phys. 84, 2325 (1986).

[38] M. M. Rudek, J. A. Fisk, V. M. Chakarov, and J. L. Katz, J. Chem. Phys. 105, 4707 (1996).

[39] J. Wölk and R. Strey, J. Phys. Chem. B 105, 11683 (2001).

[40] D. Brus, V. Ždímal, and J. Smolík, J. Chem. Phys. 129, 174501 (2008).

[41] V. B. Mikheev, P. M. Irving, N. S. Laulainen, S. E. Barlow, and V. V. Pervukhin, J. Chem. Phys. 116, 10772 (2002).

[42] D. W. Oxtoby, Acc. Chem. Res. 31, 91 (1998).

[43] D. Kashchiev, J. Chem. Phys. 118, 1837 (2003).

[44] V. G. Baidakov and K. S. Bobrov, J. Chem. Phys. 140, 184506 (2014).

[45] A. Malijevský and G. Jackson, J. Phys. Condens. Matter 24, 464121 (2012).

[46] L. S. Bartell, J. Phys. Chem. B 105, 11615 (2001).

[47] E. M. Blokhuis and J. Kuipers, J. Chem. Phys. 124, 074701 (2006).

[48] M. Iwamatsu, J. Phys. Condens. Matter 6, L173 (1994).

[49] E. M. Blokhuis and A. E. van Giessen, J. Phys. Condens. Matter 25, 225003 (2013).

[50] W. Helfrich, Z. Naturforsch. C 28, 693 (1973).

[51] M. A. González, G. Menzl, J. L. Aragones, P. Geiger, F. Caupin, J. L. F. Abascal, C. Dellago, and C. Valeriani, J. Chem. Phys. 141, 18C511 (2014). 